\title{
Decentralized Model Predictive Control of Drinking Water Networks using an Automatic Subsystem Decomposition Approach
}

\author{
Davide Barcelli * Carlos Ocampo-Martinez ${ }^{* *}$ Vicenç Puig ** \\ Alberto Bemporad ${ }^{* * *}$ \\ * Dipartimento di Ingegneria dell'Informazione, University of Siena \\ Via Roma 56, Siena 53100, Italy \\ barcelli@dii.unisi.it \\ ** Institut de Robòtica i Informàtica Industrial, CSIC-UPC \\ C/. Llorens i Artigas, 4-6, 08028 Barcelona, Spain \\ [cocampo, vpuig] @iri.upc. edu \\ *** Department of Mechanical and Structural Engineering, University \\ of Trento, Via Mesiano 77, 38100 Trento, Italy \\ bemporad@ing.unitn.it
}

\begin{abstract}
This paper proposes an automatic model decomposition approach for decentralized model predictive control (DMPC) of drinking water networks (DWNs). For a given DWN, the proposed algorithm partitions the network in a set of subnetworks by taking advantage of the topology of the network, of the information about the use of actuators, and of system management heuristics. The derived suboptimal DMPC strategy results in a hierarchical solution with a set of MPC controllers used for each partition. A comparative study between centralized MPC (CMPC) and DMPC approaches is described for the considered case study, which consists of an aggregate version of the Barcelona DWN. Results on several simulation scenarios show the effectiveness of the proposed DMPC approach in terms of the reduced computation burden and, at the same time, of the admissible lost of performance.
\end{abstract}

Keywords: Model predictive control, networked systems partitioning, hierarchical control, decentralized control, drinking water networks

\section{INTRODUCTION}

Optimization of drinking water networks (DWN) has gained much attention in the last few decades, as drinking water management in growing urban areas became an increasing concern. Limited water supplies, conservation and sustainability policies, infrastructure complexity, and consumer demand satisfaction by appropriate flow, pressure and quality levels make water management a very challenging control problem. Decision support systems provide useful guidance for human operators in complex networks, where "best" actions in resource management are not intuitive. Optimization and optimal/predictive control techniques provide important quantitative strategies for smart management of DWNs, see Brdys and Ulanicki (1994), Tu et al. (2005), among others.

Research in this field is spurred by the complexities associated with the connection management of multiple interconnected reservoirs in case of large-scale networks, which

\footnotetext{
1 This work has been partialy supported by the CICYT Ref. DPI2009-13744 of the Spanish Science and Technology Ministry, the Juan de la Cierva Research Programme (ref. JCI-20082438), the DGR of Generalitat de Catalunya (SAC group Ref. 2009/SGR/1491), and the European project WIDE, contract number FP7-IST-224168.
}

still exceeds the capabilities of existing optimization tools in finding optimal actions in a sufficiently small computation time. Mathematical programming techniques are one of the many available tools and most widely used. Their main objective consists in generating control strategies ahead in time, using techniques such as model predictive control (MPC), to guarantee a competent network service and a certain degree of reliability in probability, while simultaneously achieving certain objectives as minimization of supply and pumping costs, maximisation of water quality and leak prevention, among others. This optimization problem is usually large and nonlinear, because of the characteristics of pumps, pipeline pressure, and performances indices. So far, the aforementioned control methods for water systems based on MPC and dynamic optimization techniques have been implemented in a centralized manner over SCADA systems using a traditional hierarchical management architecture placed above the process instrumentation and basic regulatory control layers. However, such a centralized architecture leads to implementation problems because of dimensionality, multi-time scales, and spatial distribution of DWNs. The complexity of the underlying optimization problem is not the only obstacle to the implementation of centralized architectures. The main hurdle for plant-wide centralized control is that it 
is not scalable: it requires a huge model, which needs to be maintained at every change (even tiny) of the topological configuration. As a consequence, the overall complex controller may require a complete re-tuning, due to dynamical interactions. Very frequently, the costs of setting up and maintaining such a monolithic solution are prohibitive. Moreover, any maintenance operation over even a single controlled element, which of course implies turning off that element, would require a change in the complex centralized model. Then, either one ignores the element under maintenance (or simply that is temporary unavailable) with all consequent implications, or switches off the whole control system considering the availability of several control configurations.

A way of circumventing these issues is to look into decentralized model predictive control (DMPC) or distributed MPC techniques, where networked local MPC controllers are in charge of the control of actuators relative to a part of the entire water network. A few works have recently been published in the field of decentralized and distributed MPC, see Negenborn et al. (2008), Scattolini (2009), among others. The main difference between decentralized and distributed MPC is that the latter requires negotiations and re-computations of local control actions within the sampling period to increase the level of cooperation. In Barcelli and Bemporad (2009) and references therein, a decentralized MPC approach with sufficient stability criteria was proposed, which also handles the case of intermittent feedback due for instance to unreliable wireless communication.

The main contribution of this paper is an automatic subsystem decomposition approach for DMPC of DWNs. The resulting decentralized MPC controller reduces the computation burden with respect to the centralized counterpart, still maintaining a convenient level of suboptimality with respect to the control objectives. A real case study based on the Barcelona DWN is used in this paper to test the proposed methodology, showing very promising results. Theoretical investigations of stability and feasibility issues of the proposed DMPC control scheme will be addressed in future research.

\section{DWN CONTROL-ORIENTED MODELLING PRINCIPLES}

Control-oriented modelling principles for DWNs have been widely presented in the literature, see Brdys and Ulanicki (1994); Ocampo-Martinez et al. (2009). In order to obtain a control-oriented model of the DWN, the constitutive network elements as well as their basic relationships should be discussed.

Let us consider the main physical constraints of a DWN system related to the tank volumes and manipulated flows. For the case of tank volumes, the physical constraint related to the range of volume capacities for the $i$-th tank is expressed as

$$
x_{i}^{\min } \leq x_{i}(k) \leq x_{i}^{\max },
$$

where $x_{i}^{\min }$ and $x_{i}^{\max }$ denote the minimum and the maximum volume capacity, respectively, given in $\mathrm{m}^{3}$. On the other hand, the physical constraints related to manipulated flows through the system actuators are expressed as

$$
u_{i}^{\min } \leq u_{i}(k) \leq u_{i}^{\max }
$$

where $u_{i}^{\min }$ and $u_{i}^{\max }$ denote the minimum and the maximum flow capacity, respectively, given in $\mathrm{m}^{3} / \mathrm{s}$.

By considering the mass balance in the tanks, the controloriented model of a DWN in discrete-time state-space form can be written as

$$
x(k+1)=A x(k)+B u(k)+B_{p} d(k),
$$

where $x \in \mathbb{R}^{n}$ is the state vector corresponding to the water volumes of the $n$ tanks, $u \in \mathbb{R}^{m}$ represents the vector of manipulated flows through the $m$ actuators (pumps and valves), and $d \in \mathbb{R}^{p}$ corresponds to the vector of the $p$ water demands (sectors of consume). $A, B$, and $B_{p}$ are system matrices of suitable dimensions. Since the demands can be forecasted and they are assumed to be known, $d$ is a known vector containing the measured disturbances affecting the system. By also including static relations at network nodes, model (3) can be further rewritten as

$$
\begin{aligned}
x(k+1) & =A x(k)+\Gamma v(k), \\
E_{1} v(k) & =E_{2},
\end{aligned}
$$

where $\Gamma=\left[\begin{array}{ll}B & B p\end{array}\right], v(k)=\left[\begin{array}{ll}u(k)^{T} & d(k)^{T}\end{array}\right]^{T}$, and $E_{1}$, $E_{2}$ are matrices of suitable dimensions dictated by the network topology.

\section{MODEL PREDICTIVE CONTROL OF DWN}

MPC is one of the most effective and accepted strategies for control of large-scale multivariable systems (see, e.g., Maciejowski (2002)). In control of DWNs, MPC is required to compute, in a predictive way, the proper water flows that achieve the optimal performance of the network according to a given set of control objectives. MPC strategies have some important features to deal with DWNs such as the amenability for including disturbance forecasts (water demands), physical constraints, and multivariable system dynamics and objectiveswithin the control problem in a relatively simple fashion. This section describes the main ideas of the DWN control within the MPC framework, in accordance with the following operational objectives (Ocampo-Martinez et al., 2009, 2010):

Minimizing water production and transport cost. The main economic costs associated with drinking water production (treatment) are due to chemicals, legal canons, and electricity costs. The corresponding performance figure to be minimized is expressed as

$$
f_{1}(k)=W_{\alpha}\left(\alpha_{1} u(k)+\alpha_{2}(k) u(k)\right),
$$

where $\alpha_{1}$ corresponds to a known vector related to the economic costs of the water according to the selected source (treatment plant, dwell, etc.), and $\alpha_{2}(k)$ is associated with the economic cost of the flow through certain actuators (pumps only) and their control cost (pumping). Note the time-variance of $\alpha_{2}$, due to the fact that pumping efforts have different values according to the time of the day (electricity costs). $W_{\alpha}$ is the associated weight matrix of suitable dimensions.

Safety storage term. The satisfaction of water demands should be fulfilled at every time instant. However, some risk prevention mechanisms should be introduced in the 
tank management so that, additionally, the stored volume is preferably maintained around a given safety value in case of unpredicted emergency, and to guarantee future water availability in case of inaccurate demand forecasts. A quadratic expression for this concept is used and written as follows:

$$
f_{2}(k)=\left(x(k)-\beta x^{\max }\right)^{T} W_{x}\left(x(k)-\beta x^{\max }\right),
$$

where $\beta$ is a term which determines the security volume to be considered for the control law computation and matrix $W_{x}$ defines the weight of the objective in the cost function.

Smoothness of control actions. To smooth out the control action of MPC, we include the following third term in the objective function to penalize variations $\Delta u(k)=$ $u(k)-u(k-1)$ of the control signal between consecutive sampling intervals

$$
f_{3}(k)=\Delta u(k)^{T} W_{u} \Delta u(k),
$$

where $W_{u}$ is a $m \times m$ weight matrix.

In conclusion, the overall performance function $J(k)$ merging the above multiple objectives is defined as

$$
J(k)=\sum_{i=0}^{H_{u}-1} f_{1}(k+i \mid k)+\sum_{i=1}^{H_{p}} f_{2}(k+i \mid k)+\sum_{i=0}^{H_{u}-1} f_{3}(k+i \mid k),
$$

where $H_{p}$ and $H_{u}$ correspond to the prediction and control horizons, respectively. In (8), index $k$ represents the current time instant while index $i$ represents the predicted time along the horizon.

The weights are chosen in a way that the highest priority objective is the economic cost, which should be minimized while obtaining acceptable satisfaction of security and stability objectives. Collecting the parts described in previous subsections, the MPC design follows the traditional methodology (see, e.g., Maciejowski (2002)), consisting in an optimization problem where the cost function (8) is minimized subject to (1), (2) and (4). Once the minimization is performed, a vector of control actions over a given horizon is obtained. Only the first component of that vector is applied to the plant. The procedure is repeated at the next time instant, taking into account the feedback measurements coming from the system.

\section{DWN PARTITIONING APPROACH}

The application of DMPC to DWN depends crucially on how the network is decomposed into subsystems. Identifying subsystems is not an easy task in a large-scale network. The partitioning algorithm proposed in this paper aims to do this decomposition automatically by identifying clusters of elements that are weakly interconnected, in order to represent the whole network as a set of loosely coupled subsystems (see Barcelli (2008)).

\subsection{Partitioning algorithm}

As a starting point, the partitioning algorithm requires the following information of the DWN:

(i) The interconnection structure characterized by the following matrix

$$
M=\left[\begin{array}{ll}
A_{s p} & B_{s p}
\end{array}\right]
$$

where

$$
A_{s p}=\left[\begin{array}{cc}
A & 0 \\
0 & 0
\end{array}\right], \quad B_{s p}=\left[\begin{array}{c}
B \\
E
\end{array}\right]
$$

In (9), $A$ and $B$ are the system matrices in (3), subscript $s p$ identifies the matrices used for system decomposition, and $E \triangleq\left[\begin{array}{ll}E_{1} & E_{2}\end{array}\right]$ is the matrix related to the equality constraints (4b). In order to take into account different bounds on the inputs, new normalized inputs are introduced $\bar{u} \triangleq u / u_{\max }$ such that the bounds range in the interval $[0,1]$. Thus, new matrices $\bar{B}$ and $\bar{E}$ are introduced in (9b) to take into the rescaling. From matrix $M$, the adjacency matrix $E$ of the network graph can be obtained by replacing the non-zero elements by ones, leaving the zero elements unchanged.

(ii) A threshold value $\varepsilon$ that takes into account the actuator capacity of linking two tanks / nodes that, together with the actuator usage level, are used by the partitioning algorithm in order to neglect some actuators that have less effect on the behaviour of the entire system.

The partitioning algorithm proceeds by decomposing matrix $M$ into a set of submatrices, named as partitions, $P_{\varepsilon}=\left\{M_{1}, \cdots, M_{n_{p}}\right\}$ such that the edges interconnecting the subsystems correspond to elements of $M$ with magnitude no larger than $\varepsilon$. That is, the idea behind the partitioning approach is to neglect less important elements (i.e., links) in matrix $M$ such that the resulting $\tilde{M}$ is less coupled. Ideally, $\tilde{M}$ should lead to a permutation matrix $P$ such that $P^{\prime} \tilde{M} P$ is block-diagonal. This procedure is repeated iteratively by reducing $\varepsilon$ until all tanks and nodes have been assigned to a partition. Algorithm 1 summarizes the steps of the proposed partitioning algorithm.

In the first iteration, Algorithm 1 neglects a high number of elements of $M$, considerably reducing the matrix connectivity degree and obtaining a subsystem decomposition via finding a suitable $P$ that block-diagonalizes the matrix $P^{\prime} \tilde{M} P$. Every subsystem is composed by sets of state and input variables that are linked, meaning that are in the same block in the diagonal of $P^{\prime} \tilde{M} P$. Let $\mathbb{X}^{i}$ and $\mathbb{U}^{i}$ be respectively the sets of state and input variables assigned to subsystem $i$, while $L\left(\mathbb{X}^{i}\right)$ and $L\left(\mathbb{U}^{i}\right)$ determine the number of variables for each set. A subsystem is created if both numbers are different than zero. All state and input variables that are not assigned to any of the currently created subsystems, i.e., that does not belong to $\mathbb{X}^{i}$ or $\mathbb{U}^{i}$, respectively, are available for the next iteration. Otherwise, variables already assigned to a subsystem, in the current or in a previous iteration, are masked ${ }^{2}$ to prevent their reassignment to other subsystem.

Then, a new iteration of the algorithm starts by decreasing $\varepsilon$ (e.g., halving $\varepsilon$ ). Algorithm 1 iterates until all state variables are assigned to a subsystem. Note that at the next iteration the threshold decrease the set of non zero elements of $\tilde{M}$ is increased implying the new partitions to comprehend the old ones in case those were not masked.

A few remarks on the above algorithm. At any iteration of Algorithm 1, the numerical value of $\varepsilon$ is a crucial tuning

\footnotetext{
2 Let us consider a variable to be masked when it does not belong to any set since it has already been classified in a previous iteration, i.e. it is not considered in the ongoing partitioning iteration.
} 


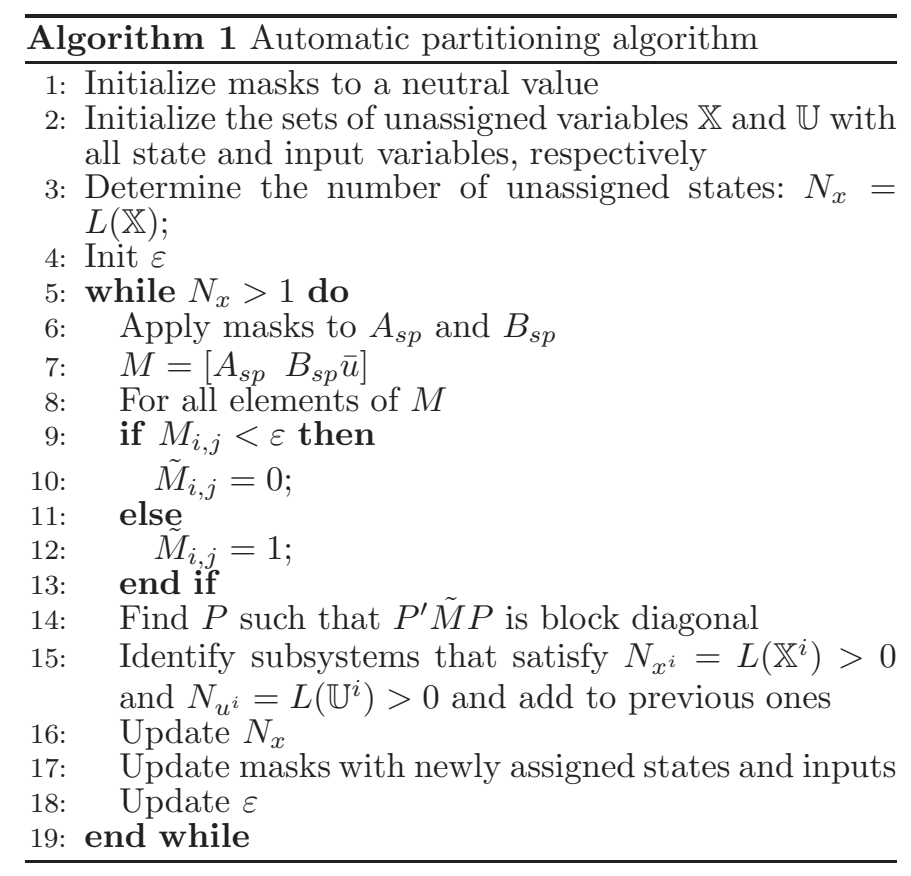

knob of the approach. A guideline is that the larger is the decreasing step, the larger is the size of the obtained subsystems. Ways for automatically determining the step size are a subject of current research.

Matrix $E$ in (9b) defines a constraint among actuators that can be easily taken into account if all the actuators belong to the same subsystem. Otherwise, since each controller manipulates every partition independently of the others, negotiations between controllers would be required to guarantee the fulfillment of node constraints.

The use of masks to prevent state reassignment avoids that submodels have overlapping states and inputs: if a state variable is used in a model by a controller, no other controller can use it. The main benefit of this choice is the very low level of coupling between partitions, but the price to pay is a potential decrease of closed-loop performance.

The current structure of the algorithm is unsuitable to handle state overlaps because it relies on links between elements that present different degree of coupling. Hence, once the stronger couplings are eliminated (using masking), the weaker ones gain relative importance. State overlaps may be introduced a posteriori based on engineering insight, in order to let the assembling of the partitions behave more likely the original centralized model. Handling overlapping in an automatic way is also a current research topic.

In some cases even relatively small connections, i.e., capable of carrying a minor amount of water per time, are very important for demand satisfaction. A way of accounting for such an issue is to perform a simulation using, for instance, a centralized MPC controller, and compute the average percentage of use for each actuator. Thus, this information could be used to weight $\bar{u}$ component-wise. The main drawback of this approach is the need of (and dependence on) simulation.

Note that the proposed algorithm can be customized by setting different importance levels of states vs. inputs, by weighting the related components in $M$. By defining

$$
M=\left[W_{A}^{\prime} A_{s p} W_{A} \quad W_{B}^{\prime} B_{s p} W_{B} \quad W_{u}^{\prime} \bar{u} W_{u}\right],
$$

where $W_{A}, W_{B}$ and $W_{u}$ are weights respectively of $A, B$ and $u$, it is possible to affect the resulting partitioning outcome.

The decomposition process of matrix $M$ reported here is similar to the one proposed by the $\varepsilon$-decomposition method in Sezer and Siljak (1986). The underlying idea in both cases is to disconnect those actuators corresponding to interconnections with strength smaller than the prescribed $\varepsilon$, identifying the disconnected subsystems. According to Sezer and Siljak (1986), there are $s$ different $\varepsilon$ decompositions $P_{\varepsilon}$ that can be obtained for different values of $\varepsilon$ satisfying

$$
\max _{i \neq j}\left|m_{i j}\right|=\varepsilon_{1}<\varepsilon_{2}<\cdots<\varepsilon_{K}=0,
$$

with $K \leq s$, where $s=\operatorname{dim}(M)$. Moreover, such decompositions are nested, that is, the partitions obtained satisfy: $P_{\varepsilon_{1}} \subset P_{\varepsilon_{2}} \cdots P_{\varepsilon_{K}}$ with $P_{\varepsilon_{1}}$ being the finest and $P_{\varepsilon_{1}}$ the coarsest. The main novelty of the algorithm presented in this paper is the matrix normalization taking into account actuator physical/operative limits, and the iterative threshold updating that allows one to take into account weaker coupling without being influenced by the stronger ones.

\section{APPLICATION DESCRIPTION AND RESULTS}

\subsection{Case-study description}

This paper considers an aggregate version of the Barcelona DWN, which is a representative version of the entire network. Here, some consumer demand sectors of the network are concentrated in a single point. Similarly, some tanks are aggregated in a single element and the respective actuators are considered as a single pumping station or valve. The complete description of this case study can be found in Ocampo-Martinez et al. (2009).

\subsection{Simulation scenarios and MPC tuning}

Demand data correspond to the consumption of drinking water of the city of Barcelona during the year 2007. Using this information, some scenarios are considered by modifying some controller parameters presented in Section 3 . They are the safety volume, denoted as $\beta$, and the weight matrices in the cost function (8). Regarding $\beta$, this parameter has been set in the following values:

(i) the $80 \%$ of $x^{\max }$, that is denoted as $\mu=0.8 x^{\max }$. This value is purely illustrative to show the effectiveness of the MPC controller;

(ii) the minimum tank volumes requested to satisfy the demands (except for tanks $x_{5}, x_{6}$ and $x_{8}$ in Figure 1, since they are considered as sources due to their strategical management requirements and network location). This second vector of safety volumes, denoted as $\eta$, is more convenient since it keeps the volumes of the tanks as low as possible, satisfying the demands at each time instant. These minimum volumes are taken from previous studies reported in Caini et al. (2009).

Let $\left(\omega_{x}, \omega_{\Delta u}\right)$ be the pair of weights associated with the weight matrices $W_{x}=\omega_{x} I$ and $W_{\Delta u}=\omega_{\Delta u} I$ used in (6) 


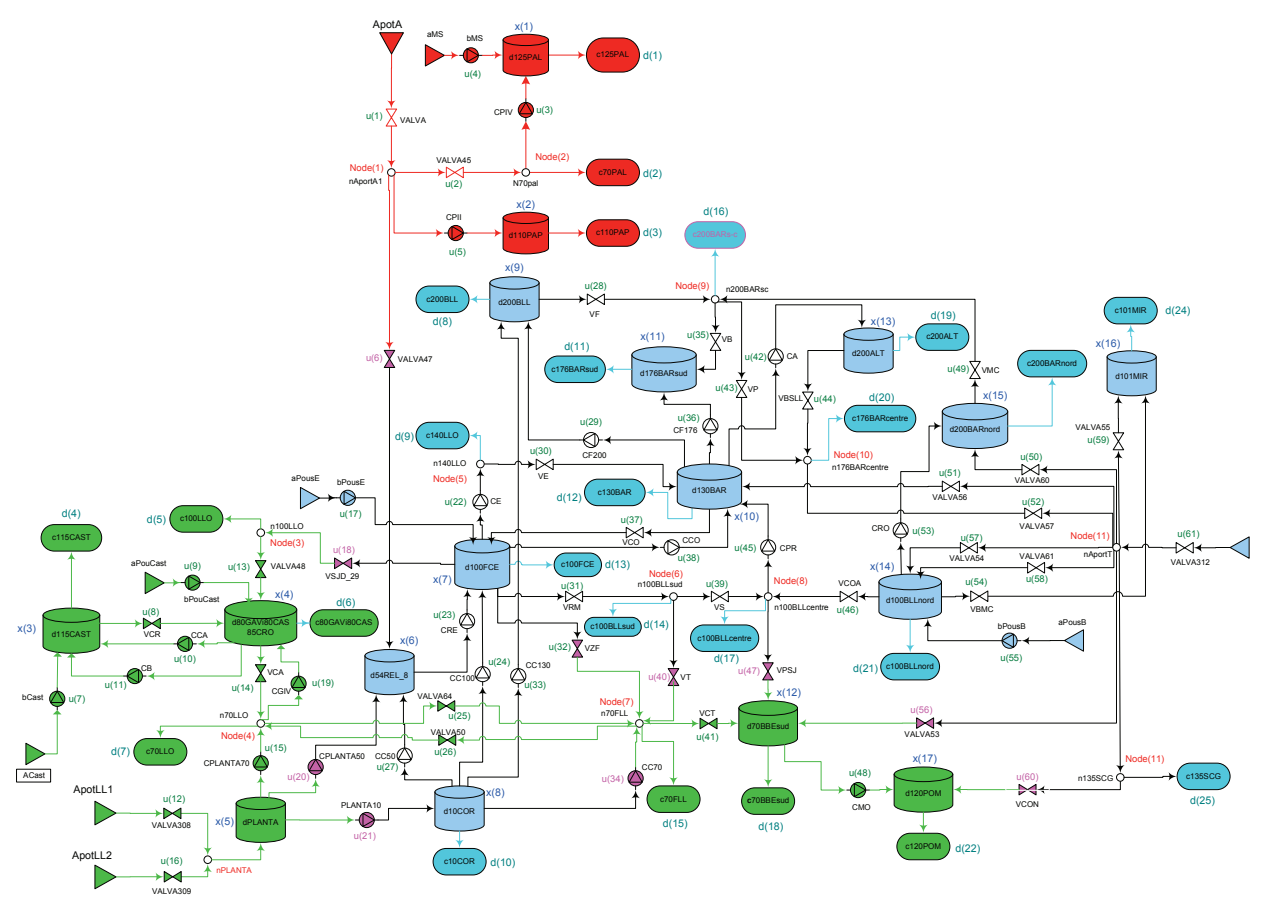

Fig. 1. Aggregate model of the Barcelona Drinking Water Network

and in (7), respectively ${ }^{3}$. We use here two pairs of weights: $(1,1)$ and $(1,0.1)$. These particular values of the weights are carefully selected, according to a previous study based on trial and error tuning procedure (Caini et al., 2009), and correspond to a couple of different prioritization of the control objectives for the particular case study.

Hence, the following scenarios have been defined:

- Scenario 1: $\beta=\mu$ and $\left(\omega_{x}, \omega_{\Delta u}\right)=(1,1)$;

- Scenario 2: $\beta=\mu$ and $\left(\omega_{x}, \omega_{\Delta u}\right)=(1,0.1)$;

- Scenario 3: $\beta=\eta$ and $\left(\omega_{x}, \omega_{\Delta u}\right)=(1,1)$;

- Scenario 4: $\beta=\eta$ and $\left(\omega_{x}, \omega_{\Delta u}\right)=(1,0.1)$.

\subsection{Partitioning of the Barcelona DWN}

Using the partitioning algorithm presented in Section 4, the Barcelona DWN is partitioned in three subsystems, as depicted in Figure 1 in different colours. The partition follows the scheme shown in Figure 2. The sizes of the resultant subsystems are defined in Table 1 . Those subsystems are composed by the following elements:

(1) Subsystem 1: composed by tanks $x_{i}, i \in\{1,2\}$, inputs $u_{j}, j \in\{1, \ldots, 5\}$, demands $d_{l}, l \in\{1,2,3\}$, and nodes $n_{q}, q \in\{1,2\}$. It is represented in Figure 1 with red colour.

(2) Subsystem 2: composed by tanks $x_{i}, i \in\{3,4,5,12$, $17\}$, inputs $u_{j}, j \in\{7, \ldots, 16,18,19,25,26,32,34,40$, $41,47,48,56,60\}$, demands $d_{l}, l \in\{4, \ldots, 7,15,18,22\}$, and nodes $n_{q}, q \in\{3,4,7\}$. It is represented in Figure 1 with green colour.

3 Note that the MPC controllers designed in the case study of this paper does not include the economic costs $\left(f_{1}\right)$ in the control objective (8). In any case, this will not change the results of the partitioning algorithm
Table 1. Dimension comparison of subystems

\begin{tabular}{ccccc}
\hline Elements & Subsys 1 & Subsys 2 & Subsys 3 & Whole Network \\
\hline Tanks & 2 & 5 & 10 & 17 \\
Actuators & 5 & 22 & 34 & 61 \\
Demands & 4 & 9 & 22 & 25 \\
Nodes & 2 & 3 & 6 & 11 \\
\hline
\end{tabular}

(3) Subsystem 3: composed by tanks $x_{i}, i \in\{6, \ldots, 11$, $13, \ldots, 16\}$, the inputs $u_{j}, j \in\{6,17,20, \ldots, 24,27, \ldots$, $31,33,35, \ldots, 39,42, \ldots, 46,49, \ldots, 55,57,58,59,61\}$, demands $d_{l}, l \in\{8, \ldots, 14,16,17,19,20,21,23,24,25\}$, and nodes $n_{q}, q \in\{5,6,8, \ldots, 11\}$. It is represented in Figure 1 with blue colour.

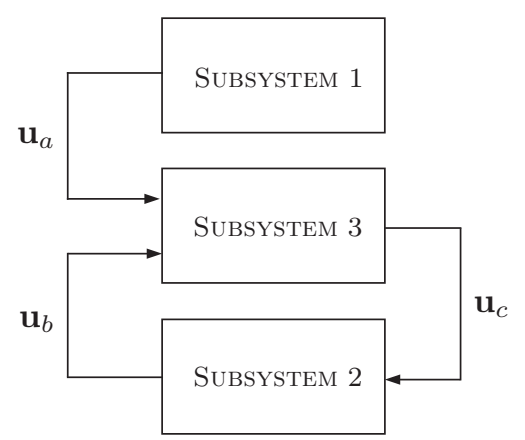

Fig. 2. Conceptual scheme of the case study partitioned in three sub-networks.

According also to the scheme in Figure 2, vectors $\mathbf{u}_{a}, \mathbf{u}_{b}$ and $\mathbf{u}_{c}$ with the shared control variables are defined as

$$
\begin{aligned}
& \mathbf{u}_{a}=u_{6}, \quad \mathbf{u}_{b}=\left[u_{20}, u_{21}\right]^{T}, \\
& \mathbf{u}_{c}=\left[u_{18}, u_{32}, u_{34}, u_{40}, u_{47}, u_{56}, u_{60}\right]^{T} .
\end{aligned}
$$


Table 2. Cost results obtained for the considered control objective and scenarios

\begin{tabular}{ccccc}
\hline SCEnARIO & Centralized MPC & \multicolumn{2}{c}{ Decentralized MPC } \\
& $\sum f_{i}$ & Cost & $\sum f_{i}$ & Cost \\
\hline SCEnARIO 1 & 58.0787 & 220.08 & 59.9548 & $223.49(1.55 \%)$ \\
SCEnARIO 2 & 57.5404 & 219.73 & 59.1040 & $223.90(1.90 \%)$ \\
SCENARIO 3 & 74.0044 & 197.85 & 76.1662 & $220.21(11.30 \%)$ \\
SCENARIO 4 & 74.3957 & 199.12 & 78.4981 & $200.43(0.66 \%)$ \\
\hline
\end{tabular}

Table 3. Time results obtained for the considered control objective and scenarios (all in s)

\begin{tabular}{ccccc}
\hline SCEnARIO & \multicolumn{2}{c}{ Centralized MPC } & \multicolumn{2}{c}{ Decentralized MPC } \\
& Total time & Max time & Total time & Max time \\
\hline SCENARIO 1 & 207.12 & 6.0866 & 128.2828 & 3.2086 \\
SCENARIO 2 & 206.27 & 7.0348 & 130.888 & 3.3209 \\
SCENARIO 3 & 210.57 & 4.9057 & 125.5362 & 4.7260 \\
SCENARIO 4 & 211.18 & 5.5524 & 126.0275 & 2.8945 \\
\hline
\end{tabular}

\subsection{Application of a hierarchical DMPC approach}

Since the obtained Barcelona DWN partitions share some control variables, the hierarchical DMPC approach described in Ocampo-Martinez et al. (2010) is applied. This hierarchical-based approach consists in defining sets of shared variables (control inputs) depending on their connection direction, i.e., if the control flow goes from a Partition $A$ to a Partition $B$ or vice versa. Once these sets are defined, it is necessary to determine the partition with the higher amount of incoming and out-coming connections. This fact locates that partition at the top of the hierarchical pyramid. Next, other partitions with less connections with respect to the latter are defined and the criterion is again applied for the following partition. Notice that, from now on, two or more partitions can be located below the one in the top, fact that defines the hierarchical pyramid.

\subsection{Results discussion}

The proposed hierarchical DMPC approach is compared with a CMPC scheme employing the simulation scenarios described in Section 5.2. The control objectives values obtained using both controllers as well as the computational times are presented in Tables 2 and 3. Moreover, the economical cost has been evaluated even if both controllers do not optimize this term. This cost has been evaluated using a water network simulation environment developed in MATLAB/SIMULINK ${ }^{\circledR}$ in Caini et al. (2009). The first column of these tables indicate the name of the considered scenario. Table 2 shows that the loss of performance is small in all scenarios. Moreover, it can be noticed from Table 3 that the DMPC controller requires about half the computation time of the CMPC controller to solve one iteration in the worst case. Thus, despite the DMPC approach leads inevitably to a small loss of performance, the benefits in terms of time and computational load are remarkable. Because of confidentiality reasons, Tables 2 and 3 show economical costs expressed in economical units (e.u.) instead of real values (Euro).

\section{CONCLUDING REMARKS}

This paper has proposed an automatic subsystem decomposition approach for decentralized model predictive control (DMPC) of drinking water networks (DWN). A hierarchical structure related to the order of execution of the DMPC controllers allows one to take into account global network constraints. A comparative study between the CMPC and DMPC approaches has been developed using as case study the aggregate model of the Barcelona DWN. Results have shown that the partition algorithm, helped by an analysis of the system topology and heuristics, yields a proper segmentation of the whole network without overlapping models. The performances of CMPC and DMPC schemes were compared in terms of economical benefits and computation demand. Results have shown the effectiveness of the DMPC strategy in the important reduction of the computation burden despite the lost of performance of the control scheme, which in turn, has resulted to be quite small.

\section{REFERENCES}

D. Barcelli. Decomposizione ottima e controllo predittivo distribuito della rete idrica di Barcellona. Master's thesis, UNISI - UPC, 2008.

D. Barcelli and A. Bemporad. Decentralized model predictive control of dynamically-coupled linear systems: Tracking under packet loss. In Proceedings of the IFAC Workshop on Estimation and Control of Networked Systems, pages 204-209, Venice (Italy), 2009.

M. Brdys and B. Ulanicki. Operational Control of Water Systems: Structures, algorithms and applications. Prentice Hall International, UK, 1994.

E. Caini, V. Puig, and G. Cembrano. Development of a simulation environment for drinking water networks: Application to centralized MPC for the Barcelona case study. Technical report, IRI (CSIC-UPC), 2009.

J.M. Maciejowski. Predictive Control with Constraints. Prentice Hall, Great Britain, 2002.

R.R. Negenborn, B. De Schutter, and J. Hellendoorn. Multi-agent model predictive control for transportation networks: Serial vs. parallel schemes. Engineering Applications of Artificial Intelligence, 21(3):353-366, 2008.

C. Ocampo-Martinez, V. Puig, G. Cembrano, R. Creus, and M. Minoves. Improving water management efficiency by using optimization-based control strategies: the Barcelona case study. Water Science \& Technology: Water supply, 9(5):565-575, 2009.

C. Ocampo-Martinez, V. Fambrini, D. Barcelli, and V. Puig. Model predictive control of drinking water networks: A hierarchical and decentralized approach. In Proceedings of the American Control Conference, Baltimore (USA), 2010.

R. Scattolini. Architectures for distributed and hierarchical Model Predictive Control: A review. Journal of Process Control, 19(5):723-731, May 2009.

M. E. Sezer and D.D. Siljak. Nested $\varepsilon$-decomposition and clustering of complex systems. Automatica, 22(3):321331, 1986.

M.Y. Tu, F. Tsai, and W. Yeh. Optimization of water distribution and water quality by hybrid genetic algorithm. Journal of Water Resources Planning and Management, 131(6):431-440, 2005. 